\title{
An Improved Finite Element Method for Solving a Kind of Nonlinear Volterra-Fredholm Integral Equation
}

\author{
Zhiguang Xiong, ${ }^{1, *}$, Kang Deng ${ }^{1}$ and Qisheng Wang ${ }^{2}$ \\ ${ }^{1}$ School of Mathematics and Computational Science, Hunan University of Science and Technology, Xiangtan, 411201, Hunan, \\ China \\ ${ }^{2}$ School of Mathematics and Computational Science, Wuyi University Jiangmen, Guangdong 529020, P.R.China \\ ${ }^{*}$ Corresponding author
}

\begin{abstract}
In this paper we extend the idea of interpolated coefficients for a kind of nonlinear Volterra-Fredholm integral equation to the improved finite element method. we introduce this numerical approximation method and Newton iterative scheme for this integral equation. Then we derive convergence estimate for exact solution and approximation solution of the equation.
\end{abstract}

Keywords-component; nonlinear volterra-fredholm integral equations; finite element method; newton iterative method; convergence analysis

\section{INTRODUCTION}

Integral equations of Volterra-Fredholm are often involved in various fields such as physics, biology and engineering, and numerical methods for solving integral equations have been studied extensively in the literature, see [1-9] for details. The finite element method with interpolated coefficients is an economic and graceful method. This method was introduced and analyzed for semilinear parabolic problems in Zlamal [13]. Xiong at. al. put the excellent interpolating coefficients idea into the finite element method for solving nonlinear differential equations [10-12].

In this paper, we shall take the interpolating coefficients idea into numerical method of the integral equations and are concerned with the improved finite element method for solving a kind of mixed nonlinear Volterra-Fredholm integral equation as

$$
\begin{gathered}
u(x)=f(x)+\lambda_{1} \int_{a}^{x} k_{1}(x, y) F(u(y)) \mathrm{d} y \\
+\lambda_{2} \int_{a}^{b} k_{2}(x, y) G(u(y)) \mathrm{d} y \\
a \leq x \leq b
\end{gathered}
$$

where the function $f, k_{1}, k_{2}$ and $F, G$ are known continuous functions defined on $[a, b],[a b] \times[a, b]$ and $\mathbf{R}$, respectively, $u(x)$ is the unknown function, $k_{1}, k_{2} \in \mathbf{R}$ and the parameters satisfy

$$
k_{1}^{2}+k_{2}^{2} \neq 0
$$

In this paper, for some results about the convergence and stability of the present numerical method, we will assume the following conditions

(i) $k_{1}, k_{2} \in C^{1}([a, b])$ and $F, G \in C^{1}$.

(ii) $\left|k_{1}\right| \gamma_{1} M_{1}+\left|k_{2}\right| \gamma_{2} M_{2} \leq \frac{1}{b-a}$, where

$$
\gamma_{1}=\max \left|k_{i}\right|, \quad i=1,2,
$$

and

$$
M_{1}=\max \left|F^{\prime}(u)\right|, \quad M_{2}=\max \left|G^{\prime}(u)\right| .
$$

\section{METHOD OF SOLUTION}

In this section, we give a new method to compute numerical solution of mixed nonlinear Volterra-Fredholm integral equation by improved finite element method. Firstly we let $J_{h}$ be a partition of the interval $[a, b]$ such that

$$
J_{h}: a=x_{0}<x_{1}<x_{2}<\cdots x_{n}=b
$$

Assume that $J_{h}$ is quasi-uniform, i.e., there is a constant $C>0$ such that

$$
h \leq C h_{j}=C\left(x_{j}-x_{j-1}\right), \quad j=1,2, \cdots, n .
$$

Then we give respectively the approximation functions for $u(x), F(u(x)), G(u(x))$ as follows 


$$
\begin{gathered}
u(x) \approx u_{h}(x)=\sum_{j=0}^{n} u_{j} \varphi_{j}(x), \\
a \leq x \leq b, \\
F(u(x)) \approx F_{h}(u(x))=\sum_{j=0}^{n} F\left(u_{j}\right) \varphi_{j}(x), \\
a \leq x \leq b, \\
G(u(x)) \approx G_{h}(u(x))=\sum_{j=0}^{n} G\left(u_{j}\right) \varphi_{j}(x), \\
a \leq x \leq b,
\end{gathered}
$$

where $\varphi_{j}(x)$ are node basis functions defined by

$$
\begin{aligned}
\varphi_{j}(x)= \begin{cases}\frac{x-x_{j-1}}{x_{j}-x_{j-1}}, & x \in\left[x_{j-1}, x_{j}\right], \\
\frac{x_{j=1}-x}{x_{j}-x_{j-1}}, & x \in\left[x_{j}, x_{j+1}\right], \\
0, & \text { otherwise, }\end{cases} \\
\quad j=0,1,2 \cdots n .
\end{aligned}
$$

The node basis functions $\varphi_{j}(x)$ satisfy

$$
\sum_{j=0}^{n} \varphi_{j}(x)=1
$$

and

$$
0 \leq \varphi_{j}(x) \leq 1, \quad j=0,1,2 \cdots n .
$$

Substitute (2)-(4) into (1) yields

$$
\begin{aligned}
u_{h}(x) & =f(x)+\lambda_{1} \int_{a}^{x} k_{1}(x, y) F_{h}(u(y)) \mathrm{d} y \\
& +\lambda_{2} \int_{a}^{b} k_{2}(x, y) G_{h}(u(y)) \mathrm{d} y
\end{aligned}
$$

which implies

$$
\begin{gathered}
\sum_{j=0}^{n} u_{j} \varphi_{j}(x)=f(x) \\
+\lambda_{1} \sum_{j=1}^{n} F\left(u_{j}\right) \int_{a}^{x} k_{1}(x, y) \varphi_{j}(y) \mathrm{d} y \\
+\lambda_{2} \sum_{j=1}^{n} G\left(u_{j}\right) \int_{a}^{b} k_{2}(x, y) \varphi_{j}(y) \mathrm{d} y,
\end{gathered}
$$

Taking $x=x_{i}, i=1,2, \cdots, n$ in (8) respectively, we have a system of equations from functional integral equation

$$
\begin{gathered}
u_{i}=f\left(x_{i}\right) \\
+\lambda_{1} \sum_{j=1}^{i} F\left(u_{j}\right) \int_{a}^{x_{i}} k_{1}\left(x_{i}, y\right) \varphi_{j}(y) \mathrm{d} y \\
+\lambda_{2} \sum_{j=1}^{n} G\left(u_{j}\right) \int_{a}^{b} k_{2}\left(x_{i}, y\right) \varphi_{j}(y) \mathrm{d} y,
\end{gathered}
$$

where $i=1,2, \cdots, n$.

Letting

$$
\begin{aligned}
& \mathbf{A}=\left(\int_{a}^{x_{i}} k_{1}\left(x_{i}, y\right) \varphi_{j}(y) \mathrm{d} y\right)_{(n+1) \times(n+1)}, \\
& \mathbf{B}=\left(\int_{a}^{b} k_{2}\left(x_{i}, y\right) \varphi_{j}(y) \mathrm{d} y\right)_{(n+1) \times(n+1)}, \\
& \mathbf{U}=\left(u_{0}, u_{1}, \cdots, u_{n}\right)^{T}, \\
& \mathbf{f}=\left(f\left(x_{0}\right), f\left(x_{1}\right), \cdots, f\left(x_{n}\right)\right)^{T},
\end{aligned}
$$

and

$$
\begin{aligned}
& \mathbf{F}(\mathbf{U})=\left(F\left(u_{0}\right), F\left(u_{1}\right), \cdots, F\left(u_{n}\right)\right)^{T}, \\
& \mathbf{G}(\mathbf{U})=\left(G\left(u_{0}\right), G\left(u_{1}\right), \cdots, G\left(u_{n}\right)\right)^{T},
\end{aligned}
$$

the nonlinear system of (9) is rewritten in the vector form as follows

$$
\mathbf{H}(\mathbf{U})=\mathbf{U}-\mathbf{f}-\lambda_{1} \mathbf{A F}(\mathbf{U})-\lambda_{2} \mathbf{B G}(\mathbf{U})=\mathbf{0} .
$$

From (10), we obtain corresponding Newton iterative algorithm scheme

$$
\begin{gathered}
\mathbf{U}^{k+1}=\mathbf{U}^{k}-\left[\mathbf{D H}\left(\mathbf{U}^{k}\right)\right]^{-1} \mathbf{H}\left(\mathbf{U}^{k}\right), \\
k=0,1,2, \cdots
\end{gathered}
$$

where $\mathbf{D}$ denotes differential with respect to vector $\mathbf{U}$.

\section{CONVERGENCE ANALYSIS}

This section we analyze the error of the finite element method. To start our analysis, first define discrete norm by 


$$
\left\|u(x)-u_{h}\right\|_{h, \infty}=\max _{0 \leq j \leq n}\left|u\left(x_{j}\right)-u_{j}\right| .
$$

For our convergence analysis, we need a lemma as follows.

Lemma 1. Assume that $a, b>0,4 a b<1$ and $x \geq 0$, $x \leq b+a x^{2}$, then $x<a b$.

From the basic mathematical analysis, it is obvious that the lemma is established.

Now we state the convergence error estimate result as follow.

Theorem 1. Assume that $f(x)$ is a function defined on $[a, b]$, and that $F(t), G(t)$ in $R=(-\infty, \infty)$ and $k_{1}(x, t), k_{2}(x, t)$ in $[a, b] \times[a, b]$ are sufficiently smooth continuous and arbitrary differentiable, $u(x)$ is an exact solution of (1), $u_{h}(x)$ is the finite element solution of (9), then

$$
\begin{gathered}
\|e\|_{h, \infty}=\left\|u(x)-u_{h}(x)\right\|_{h, \infty} \\
\leq \frac{\frac{1}{4}\left(M_{1,2}+M_{2,2}\right) h^{2}}{1-\left(\left|\lambda_{1}\right| \gamma_{1} M_{1,1}+\left|\lambda_{2}\right| \gamma_{2} M_{2,1}\right)(b-a)} .
\end{gathered}
$$

Proof. Subtracting (7) from (1) and taking $x=x_{i}$ gives

$$
\begin{aligned}
e_{i} & =\lambda_{1} \int_{a}^{x_{i}} k_{1}\left(x_{i}, y\right)\left[F(u(y))-F_{h}(u(y))\right] \mathrm{d} y \\
& +\lambda_{2} \int_{a}^{b} k_{2}\left(x_{i}, y\right)\left[G(u(y))-G_{h}(u(y))\right] \mathrm{d} y,
\end{aligned}
$$

where $e_{i}=u\left(x_{i}\right)-u_{i}$. One can easily find that

$$
\begin{aligned}
& F(u(y))-F_{h}(u(y)) \\
= & F(u(y))-F_{n}(u(y))+F_{n}(u(y))-F_{h}(u(y)) \\
= & R_{F}+\sum_{j=0}^{n}\left[F\left(u\left(y_{j}\right)\right)-F\left(u_{j}\right)\right] \varphi_{j}(y) \\
= & R_{F}+\sum_{j=0}^{n}\left[F^{\prime}\left(u_{j}\right) e_{j}+\frac{1}{2} F^{\prime \prime}\left(\xi_{j}\right) e_{j}^{2}\right] \varphi_{j}(y),
\end{aligned}
$$

and similarly

$$
\begin{aligned}
& G(u(y))-G_{h}(u(y)) \\
= & R_{G}+\sum_{j=0}^{n}\left[G^{\prime}\left(u_{j}\right) e_{j}+\frac{1}{2} G^{\prime \prime}\left(\eta_{j}\right) e_{j}^{2}\right] \varphi_{j}(y),
\end{aligned}
$$

where

$$
F_{n}(u(y))=\sum_{j=0}^{n} F\left(u\left(y_{j}\right)\right),
$$

$$
G_{n}(u(y))=\sum_{j=0}^{n} G\left(u\left(y_{j}\right)\right)
$$

and

$$
\begin{aligned}
& R_{F}=F(u(y))-F_{n}(u(y)), \\
& R_{G}=G(u(y))-G_{n}(u(y))
\end{aligned}
$$

are the remainder of interpolation corresponding to the finite element, respectively. By use of interpolation polynomial error estimation, we have

$$
\left|R_{F}\right| \leq \frac{1}{8} M_{1,2} h^{2}, \quad\left|R_{G}\right| \leq \frac{1}{8} M_{2,2} h^{2}
$$

where

$$
\begin{aligned}
& M_{1,2}=\max \left|F^{\prime \prime}(u)\right|, \\
& M_{2,2}=\max \left|G^{\prime \prime}(u)\right| .
\end{aligned}
$$

Substituting (14)-(17) into (13), we find

$$
\begin{gathered}
\left|e_{i}\right| \leq \frac{1}{8}\left(M_{1,2}+M_{2,2}\right) h^{2} \\
+\sum_{j=1}^{n}\left[\left|\lambda_{1}\right| \gamma_{1}\left(M_{1,1}\left|e_{j}\right|+\frac{1}{2} M_{1,2}\left|e_{j}\right|^{2}\right)\right.
\end{gathered}
$$

$$
\begin{aligned}
& \left.+\left|\lambda_{2}\right| \gamma_{2}\left(M_{2,1}\left|e_{j}\right|+\frac{1}{2} M_{2,2}\left|e_{j}\right|^{2}\right)\right] \int_{a}^{b}\left|\varphi_{j}(y)\right| \mathrm{d} y \\
& \leq \frac{1}{8}\left(M_{1,2}+M_{2,2}\right) h^{2} \\
& +\left(\left|\lambda_{1}\right| \gamma_{1} M_{1,1}+\left|\lambda_{2}\right| \gamma_{2} M_{2,1}\right)(b-a)\|e\|_{h, \infty} \\
& +\frac{1}{2}\left(\left|\lambda_{1}\right| \gamma_{1} M_{1,2}+\left|\lambda_{2}\right| \gamma_{2} M_{2,2}\right)(b-a)\|e\|_{h, \infty}^{2}
\end{aligned}
$$

Using the conditions (i) and (ii), we get

$$
\begin{aligned}
\|e\|_{h, \infty} & \leq \frac{\frac{1}{8}\left(M_{1,2}+M_{2,2}\right) h^{2}}{1-\left(\left|\lambda_{1}\right| \gamma_{1} M_{1,1}+\left|\lambda_{2}\right| \gamma_{2} M_{2,1}\right)(b-a)} \\
& +\frac{\left(\left|\lambda_{1}\right| \gamma_{1} M_{1,2}+\left|\lambda_{2}\right| \gamma_{2} M_{2,2}\right)(b-a)}{2-2\left(\left|\lambda_{1}\right| \gamma_{1} M_{1,1}+\left|\lambda_{2}\right| \gamma_{2} M_{2,1}\right)(b-a)}\|e\|_{h, \infty}^{2} .
\end{aligned}
$$

Application of Lemma 1 yields the desired estimate (12) and completes the proof of Theorem 1. 


\section{ACKNOWLEDGMENT}

This work was supported by National Natural Science Foundations of China under Project (Grant No. 11571102) and the Natural Science Fundation of Guangdong Province (No.2015A030313643).

\section{REFERENCES}

[1] M. A. Abdou, Fredholm-Volterra integral equation of the first kind and contact problem, Appl. Math. Comput.,Vol.125, 2002, pp.177-193.

[2] L. K. Bieniasz, Automatic solution of integral equations describing electrochemical transients at dropping mercury electrodes. Journal of Electroanalytical Chemistry, Vol.705 ,2013,pp. 44-51.

[3] F. Bloom, Asymptotic bounds for solutions to a system of damped integro-differential equations of electromagnetic theory, J. Math. Anal. Appl., Vol.73,1980,pp. 524---542.

[4] C. H. Hsiao and D. L. Young, Calculation of hydrodynamic forces for unsteady stokes flows by singularity integral equations based on fundamental solutions. Journal of Mechanics, Vol.30,2014, pp.129-136.

[5] K. Maleknejad and Y. Mahmoudi, Taylor polynomial solution of highorder nonlinear Volterra-Fredholm integro-differential equations, Appl. Math. Comput., Vol.145,2003,pp. 641-653.

[6] K. K. Wang and Q. S. Wang, Lagrange collocation method for solving Volterra-Fredhelm integral equations, Appl. Math. Comput., Vol.219,2013,pp.10434-10440.

[7] K. K. Wang, Q. S. Wang and K. Z. Guan, Iterative method and convergence anlysis for kind of mixed nonlinear Volterra-Fredholm integral equation. Appl. Math. Comput., Vol.225,2013,pp. 631-637.

[8] K. K. Wang and Q. S. Wang, Taylorpolynomial method and error estimation for a kind of mixed Volterra-Fredholm integral equations. Appl. Math. Comput. Vol.229,2014, pp.53-59.

[9] A. M. Wazwaz, A first course in integral equations(Second edition). World Scientific Publishing Co. Pte. Ltd, 2015.

[10] Z. G. Xiong and C. M. Chen, Superconvergence of rectangular finite element with interpolated coefficients for semilinear elliptic problem, Appl. Math. Comput. Vol.181,2006, pp.1577-1584.

[11] Z. G. Xiong and Y. P. Chen, Finite volume element method with interpolated coefficients for two-point boundary value problem of semilinear differential equations, Comput. Methods Appl. Mech. Engrg., Vol.196,2007, pp.3798-3804.

[12] Z. G. Xiong and Y. P. Chen, Triangular finite volume element method with interpolated coefficients for a semilinear elliptic equation, Journal of Computational Mathematics, Vol.32(2),2014, pp.152-168.

[13] M. Zlamal, A finite element solution of the nonlinear heat equation, RAIRO Model. Anal. Numer., Vol.14, 1980,pp.203-216. 